\title{
Results of a Double-Blind, Randomized, Placebo-Controlled Phase 1 Study to Evaluate the Safety and Pharmacokinetics of Anti-Zika Virus Immunoglobulin
}

\author{
Jane White, ${ }^{1}$ Priya Tunga, ${ }^{2}$ Deborah M. Anderson, ${ }^{2}$ Ken Iledan, ${ }^{2}$ Tobi Loreth, ${ }^{2}$ Geraldine S. Parrera, ${ }^{2}$ Hugo Astacio, ${ }^{2}$ \\ Bojan Drobic, ${ }^{2}$ and Jason S. Richardson ${ }^{2 *}$ \\ ${ }^{1}$ Emergent BioSolutions Inc., Gaithersburg, Maryland; ${ }^{2}$ Emergent BioSolutions Canada Inc., Winnipeg, Manitoba, Canada
}

\begin{abstract}
Zika virus (ZIKV) is transmitted primarily through infected Aedes aegypti or Aedes albopictus mosquitoes. ZIKV infection during pregnancy was linked to adverse fetal/infant outcomes, including microcephaly, brain anomalies, ocular disorders, intrauterine growth restriction, and other congenital malformations. Human anti-Zika virus immunoglobulin (ZIKV-lg) is being developed for prophylaxis of ZIKV in at-risk populations, including women of childbearing potential and pregnant women. A phase 1 single-center, double-blind, randomized, placebo-controlled study was conducted to assess the safety and pharmacokinetics (PK) of a single 50.0-mL ZIKV-lg intravenous dose in healthy adult male or nonpregnant female subjects 18 to 55 years of age. Subjects received either ZIKV-lg $(n=19)$ or saline placebo $(n=11)$. Safety was evaluated based on adverse events (AEs), laboratory test results, physical examinations, and vital signs. Overall, there were 11 subjects (36.7\%) with treatment-related AEs including eight subjects (42.1\%) in the ZIKV-Ig group and three subjects (27.3\%) in the placebo group. Of the AEs considered treatment related, three subjects (15.8\%) experienced headache (mild). There were no serious AEs, no deaths, and no discontinuations resulting from AEs. Overall, the safety profile of ZIKV-Ig in this study population of healthy adult subjects appeared to be safe and well tolerated. The results of the pharmacokinetic analysis determined that ZIKV-Ig had a maximum observed concentration of $182.3 \mathrm{U} / \mathrm{mL}$ (coefficient of variation, $21.3 \%$ ), the time at which $\mathrm{C}_{\max }$ occurred of 2.3 hours \pm 1.0 (SD), an area under the concentration-time curve ${ }_{0-\infty}$ of $77,224 \mathrm{~h} \times \mathrm{U} / \mathrm{mL}$ (coefficient of variation, $17.9 \%$ ), and a half-life of 28.1 days, which is similar to other human-derived commercial Ig intravenous products.
\end{abstract}

\section{INTRODUCTION}

Zika virus (ZIKV) is an RNA Flavivirus related closely to Dengue virus (DENV), Yellow fever virus, Japanese encephalitis virus, and West Nile virus (WNV). ZIKV is transmitted primarily through the bite of an infected Aedes sp. mosquito (Ae. aegypti or Ae. albopictus); however, sexual transmission has also been frequently reported. ${ }^{1,2}$ Zika fever (also known as ZIKV disease) is an illness caused by this virus. Currently there are no licensed products for the prevention or treatment of ZIKV infection.

The first recorded outbreak of ZIKV disease was reported from the Island of Yap (Federated States of Micronesia) in 2007, followed by a large outbreak in French Polynesia in 2013, and other countries and territories in the Pacific. ${ }^{3}$ In 2015, Brazil reported a large outbreak, and viral transmission soon appeared throughout the Americas and other regions of the world. ${ }^{3}$ In the United States these outbreaks resulted in limited local transmission in Florida and Texas, including widespread transmission in Puerto Rico and the U.S. Virgin Islands, and an increase in travel-associated cases. ${ }^{3}$ According to the $\mathrm{WHO}$, as of July 2019,87 countries and territories have evidence of mosquito-borne transmission of ZIKV, distributed across four of the six WHO-defined regions, including the African Region, Region of the Americas, Southeast Asia Region, and Western Pacific Region. ${ }^{4,5}$ All areas with previous reports of ZIKV transmission have the potential for re-emergence. There is also the potential risk for ZIKV to spread to additional countries, as 61 countries and/or territories globally have evidence of established competent mosquito vectors but have not yet documented ZIKV

*Address correspondence to Jason S. Richardson, Emergent BioSolutions Canada Inc., 155 Innovation Dr. Winnipeg MB, Canada, R3T 5Y3. E-mail: jrichardson@ebsi.com transmission to humans. ${ }^{4,5}$ It is also possible that some of these countries have or have had transmission that has not yet been detected or reported. ${ }^{5}$

Most individuals ( $80 \%$ ) infected by ZIKV, are asymptomatic, whereas symptomatic individuals typically present with acute onset of fever, maculopapular rash, joint pain, headache, or non-purulent conjunctivitis that usually lasts from several days to a week. ${ }^{2}$ However, ZIKV infection has been linked to more severe disease outcomes such as GuillainBarré syndrome ${ }^{6,7}$ and other neurological impairments, ${ }^{8}$ albeit infrequently. In addition, ZIKV infection during pregnancy has been linked to adverse fetal/infant outcomes, including microcephaly, ${ }^{9,10}$ serious brain anomalies, ${ }^{11,12}$ ocular disorders, ${ }^{12}$ intrauterine growth restriction, and other congenital malformations resulting in congenital Zika syndrome. ${ }^{13,14}$ The percentage of fetuses/infants with possible ZIKV-associated birth defects ranges from $4 \%$ to $8 \%$, depending on maternal time of ZIKV exposure during pregnancy. ${ }^{15}$ The ability of ZIKV to infect and damage developing fetuses implies the virus can cross and/or bypass the placental barrier, but the mechanism remains unclear. ${ }^{16}$ Other flaviviruses, including DENV, are not associated with vertical transmission or congenital disorders, which suggests this mechanism may be specific to ZIKV. ${ }^{17}$

Emergent BioSolutions Canada Inc. (EBCl) developed human anti-Zika virus immunoglobulin (ZIKV-lg), a human hyperimmune product of purified gamma IgG fraction of human plasma containing polyclonal antibodies reactive to ZIKV. ZIKV-lg is prepared from pooled plasma collected at U.S. Food and Drug Administration (FDA)-licensed plasma collection centers from healthy adult donors who have elevated levels of antibodies reactive to ZIKV, and was manufactured using EBCl's hyperimmune manufacturing platform process that is used to manufacture FDA-licensed products that include $\mathrm{CNJ}-016^{\mathrm{TM}}$ (Vaccinia Immune Globulin Intravenous (Human) [VIGIV]); 
Emergent BioSolutions, Winnipeg, Canada), ANTHRASIL ${ }^{\circledR}$ (Anthrax Immune Globulin Intravenous [Human]; Emergent BioSolutions, Winnipeg, Canada), WinRho ${ }^{\circledR}$ SDF (Rho(D) Immune Globulin Intravenous [Human]), Saol Therapeutics Inc., Roswell, GA, USA), HepaGam B ${ }^{\circledR}$ (Hepatitis B Immune Globulin Intravenous [Human]), Emergent BioSolutions, Winnipeg, Canada), and VariZIG $^{\circledR}$ (Varicella Zoster Immune Globulin [Human)]; Emergent BioSolutions, Winnipeg, Canada).

In this first-in-human phase 1 clinical study (ZK-001; ClinicalTrials.gov identifier NCT03624946), the safety, tolerability, and pharmacokinetics (PK) of a 50.0-mL intravenous (IV) dose ( 50-100 mg/kg) of ZIKV-lg was assessed in healthy adults.

\section{MATERIALS AND METHODS}

Study design and participants. Ours was a randomized, double-blind, placebo-controlled single-dose study conducted at a phase 1 unit (Syneos Health) in Toronto, Ontario, Canada. The study protocol was approved by Advarra Institutional Review Board and was conducted in accordance with the International Council for Harmonisation good clinical practice guidelines and all applicable local regulatory requirements and laws. Participants provided written informed consent before any study procedures occurred and were re-consented, as necessary in the occurrence of a study protocol amendment.

Briefly, notable inclusion criteria included enrollment of healthy adult male or non-pregnant female volunteers 18 to 55 years of age who had a body mass index of 18.0 to 30.0 $\mathrm{kg} / \mathrm{m}^{2}$, and were in good health as determined by no clinically significant findings from medical history, physical examination, electrocardiogram, clinical laboratory assessments, and vital sign measurements.

Participants were excluded based on screening assessments if they were administered (or planned to use during the study) an investigational product within 30 days of screening, blood products within the 12 months up to screening, and/or live vaccines within 28 days prior to screening. Participants were excluded if they had a history of hypersensitivity to blood (or plasma) products, IgA deficiency, hypercoagulable conditions, myocardial infarction, stroke, renal impairment/ failure, a chronic or acute severe neurological condition, and/ or the opinion of the investigator that it would be unwise to allow participation of the individual in the study.

Criteria were included to ensure subjects did not have preexisting antibodies to flaviviruses to mitigate interference with the PK assessment, such as excluding individuals with a history of Flavivirus infection or previous vaccination with licensed or investigational Flavivirus vaccine. At the screening visit, a serum sample was taken to perform viral marker serology testing for ZIKV IgG and IgM antibodies, DENV IgM and IgG antibodies, and WNV IgM and IgG antibodies. In addition, a ZIKV nucleic acid test (NAT) was performed on serum and urine samples. At baseline, viral marker serology testing for ZIKV IgG, ZIKV IgM, and viral marker ZIKV NAT of serum and urine was also performed. Screening serology results for DENV and WNV were reported along with baseline ZIKV NAT results for serum and urine, and baseline ZIKV IgM and IgG serology results.

Because of the limited number of plasma donors with elevated levels of antibodies to ZIKV, donor plasma with high isoagglutinin titers (e.g., anti-A and anti-B antibodies) were not excluded from the plasma pool used for the manufacture of the ZIKV-Ig clinical lot. Therefore, for this study, ZIKV-Ig was intended for IV administration only to subjects with blood types $\mathrm{O}+$ or $\mathrm{O}$ - individuals to mitigate the occurrence of hemolytic reactions in subjects. For this reason, participants were excluded if they had blood types $A, B$, or $A B$. The full inclusion and exclusion criteria for this study is provided in Supplemental Appendix S1.

Randomization and masking/blinding. A randomization scheme was provided by an unblinded contract research organization (CRO) statistician who was not involved in study treatment administration or subject assessments. The randomization program was written and documented per the CRO's standard operating procedures and in accordance with EBCl's Development of Statistical Analysis System Programs and Good Statistical Analysis System Programming Practice standard operating procedures. Subjects were assigned randomly to receive a single dose of ZIKV-Ig or a single dose of placebo (normal saline, $0.9 \%$ sodium chloride) by IV infusion.

To maintain the blind, all study drugs (ZIKV-Ig or placebo) were dispensed in a comparable manner. The pharmacy staff assigned to the study at the CRO's phase 1 clinical site were unblinded to access the randomization assignment and prepare the study treatment. Both the site principal investigator $(\mathrm{PI})$ and subjects were masked to the treatment assignment. Staff at $\mathrm{EBCl}$ were also masked to study drug assignment throughout the study to facilitate ongoing monitoring of the safety of the subjects.

Investigational product dose. The clinical dose of ZIKVIg was $50.0 \mathrm{~mL}$ (undiluted) containing a total of $4.65 \mathrm{~g} \mathrm{lgG}$ protein $(93 \mathrm{mg} / \mathrm{mL})$. The liquid product contained 30 to $130 \mathrm{mg} / \mathrm{mL}$ protein (of which, at least $96 \%$ is purified human IgG) stabilized with $10 \%$ maltose and $0.03 \%$ polysorbate 80 . The potency of the drug product, determined by a cellbased ZIKV microneutralization assay, was $10,612 \mathrm{U} / \mathrm{mL}$. The per-subject duration of infusion was expected to be complete within 33 minutes based on a per-protocol rate of infusion $(1.0 \mathrm{~mL} / \mathrm{min}$ for the first 15 minutes with an increase to the rate to $2.0 \mathrm{~mL} / \mathrm{min}$ for the remainder of the IV infusion).

Staggered dosing schedule and safety monitoring. The first six subjects were randomized $1: 1$ in pairs in a doubleblind fashion to receive either ZIKV-Ig or placebo control. Dosing was staggered over 3 days, during which two subjects per day were randomized $1: 1$ and dosed to receive either ZIKV-Ig or placebo 3 hours apart (Figure 1). Two subjects per day were dosed at least 3 hours apart, and each subgroup (dosed at least 1 day apart) was staggered over 3 days for subgroups 1A, 1B, and $1 \mathrm{C}$. The $\mathrm{Pl}$ reviewed the available safety information, including reported adverse events (AEs) within 3 hours after study treatment administration, concomitant medications, and vital signs (15 minutes into the IV infusion, at the end of the IV infusion, and 1 hour and 3 hours after study drug administration) between group 1 subgroups. The Pl assessed the data for safety concerns after all six subjects from group 1 collectively, prior to randomization of group 2. Group 2 consisted of six subjects randomized 2:1 in a double-blind fashion to receive either ZIKV-Ig or placebo, dosed at least 30 minutes apart. An independent Safety Monitoring Committee review consisted of the PI and EBCl's blinded medical monitor (MM). The Safety Monitoring Committee reviewed safety data for the 


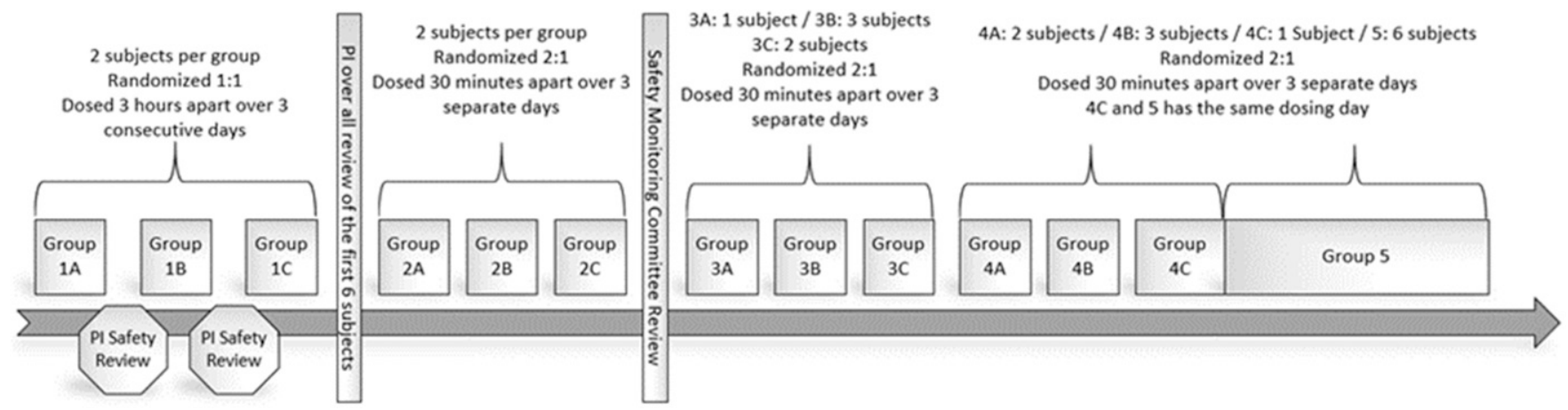

FIGURE 1. Illustrative representation of the ZK-001 treatment schedule. Group 1A $(n=2)$, group 1B ( $n=2)$, group and 1C $(n=2)$ were randomized $1: 1$ in a double-blind fashion to receive either human anti-Zika virus immunoglobulin (ZIKV-lg) or placebo. Two subjects per day were dosed at least 3 hours apart and each subgroup was dosed at least 1 day apart over 3 days, with the principal investigator (PI) safety data review occurring between subgroups followed by an overall safety data review of all 6 group 1 subjects prior to group 2 dosing. Groups $2 \mathrm{~A}(n=2), 2 \mathrm{~B}(n=2)$, and 2C $(n=2)$ were randomized 2:1 in a double-blind fashion to receive either ZIKV-lg or placebo and were dosed 30 minutes apart on three separate days. An independent Safety Monitoring Committee reviewed safety data for the first 12 dosed subjects (groups A and B) prior to group 3 dosing. Groups 3A $(n=1)$, 3B $(n=3)$, and 3C $(n=2)$ were randomized 2:1 in a double-blind fashion to receive either ZIKV-lg or placebo and were dosed 30 minutes apart on three separate days. Groups 4A $(n=2), 4 \mathrm{~B}(n=3)$, and 4C $(n=1)$ were randomized 2:1 in a double-blind fashion to receive either ZIKV-lg or placebo and were dosed 30 minutes apart on three separate days. Group $5(n=6)$ was randomized 2:1 in a double-blind fashion to receive either ZIKV-Ig or placebo and was dosed 30 minutes apart on a single day (group 4C).

first 12 dosed subjects (collected up to 72 hours post-study drug administration), prior to the remaining 18 subjects being randomized 2:1 and administered the study drug (at least 30 minutes apart) in three separate groups of six subjects (groups 3, 4, and 5).

Procedures and assessments. The schedule of events is provided in Table 1, which displays the planned clinic visits and the per-visit assessments. Briefly, this study consisted of a screening visit to establish subject eligibility (Supplemental Appendix S1) within 35 days prior to study drug administration on day 1 . Subjects arrived at the phase 1 clinic the day before (day -1) drug administration on day 1 and stayed until 24 hours after drug administration (day 2). Study assessments for safety and PK analysis were performed at the time of admission to the clinic on Day -1 , on Day 1 , predose, and post-dose at 1, 3, 8, and 24 hours (day 2 before discharge from the clinic). Subjects were scheduled to return to the phase 1 clinic for assessments on days 3, 4, 6, 8, 10, 12, $15,22,29,43$, and 57 , and at the end-of-study visit on day 85 .

Safety assessment. The primary outcome was safety, which was a descriptive comparison of incidence and severity (intensity) of any $A E$ among groups of subjects administered ZIKV-Ig versus subjects administered a placebo control for the safety population. The safety of the study drug was assessed by monitoring AEs; laboratory results for blood chemistry, hematology, vital signs, and concomitant medications collected throughout the study; as well as physical examination performed at the end-of-study visit on day 85. AEs were reported spontaneously by the subjects and/or elicited by the PI (or designee) by asking the subjects non-leading questions. All AEs were examined by the PI (or sub-investigator) for assessment of intensity as mild (awareness of a sign or symptom but subject can tolerate), moderate (discomfort enough to cause interference with normal daily activity), or severe (resulting in an inability to do work or do usual daily activity). The association of the $\mathrm{AE}$ with the study drug was assessed by the PI as "related" or "not related/no relationship to" the study drug according to International Council for Harmonisation E2A and 21.CFR.312. All AEs were classified according to the
Medical Dictionary for Regulatory Activities (v. 21.0). In addition, product class-specific AEs such as hypersensitivity (allergic reaction, anaphylaxis), acute renal dysfunction/failure, aseptic meningitis syndrome, hemolysis or hemolytic anemia, thrombotic events, or transfusion-related acute lung injury were of particular interest in this study and were considered AEs of special interest (AESI).

PK assessment. The secondary outcomes measure for this study were the PK parameters of ZIKV-Ig. A validated MAGPIX ${ }^{\circledR}$ assay targeting anti-ZIKV antibodies that bind recombinant ZIKV E-protein was used for the primary PK analysis. The MAGPIX (xPONENT software $v 4.2$, Luminex Corporation, Austin, TX) binding assay is a high-throughput method to quantify antibodies (IgG) binding to ZIKV E-protein. ZIKV E-protein antigen was bound to magnetic carboxylated beads. E-protein binding antibodies present in the diluted clinical serum samples bound to the coupled beads. A phycoerythrin conjugated goat anti-human IgG fragment crystallizable region $(\mathrm{Fc})$ antibody was added to the bound sample. The MAGPIX ${ }^{\circledR}$ instrument detected different distinct wavelengths emitted from the beads, and used them to classify the bead type and determine the median fluorescent intensity of the phycoerythrin-labeled antibody, which was proportional to the amount of bound E-protein antibodies. A seven-point calibration curve was run in each assay using the ZIKV-Ig reference standard, and sample concentrations were calculated from a five-parameter logistic standard curve generated from the calibrator concentrations.

In addition, an xCelligence functional assay (xCelligence System (RTCA software v 2.1.0 Agilent, Santa Clara, CA)) was used to quantify ZIKV neutralization activity of clinical serum samples used in support with the MAGPIX binding PK assay. VeroE6 cells were plated onto 96-well E-plates at a density of 15,000 cells/well, and were then incubated overnight to form a confluent monolayer. Heat-inactivated serum samples were diluted appropriately and incubated with a viral load equivalent to a 100-tissue culture infectious dose in which $50 \%$ of cells are infected with ZIKV (strain PRVABC59). Sample and virus mixtures were added to the cell plate then incubated to allow for non-neutralized virus 
SAFETY AND PHARMACOKINETICS OF ZIKV-IG

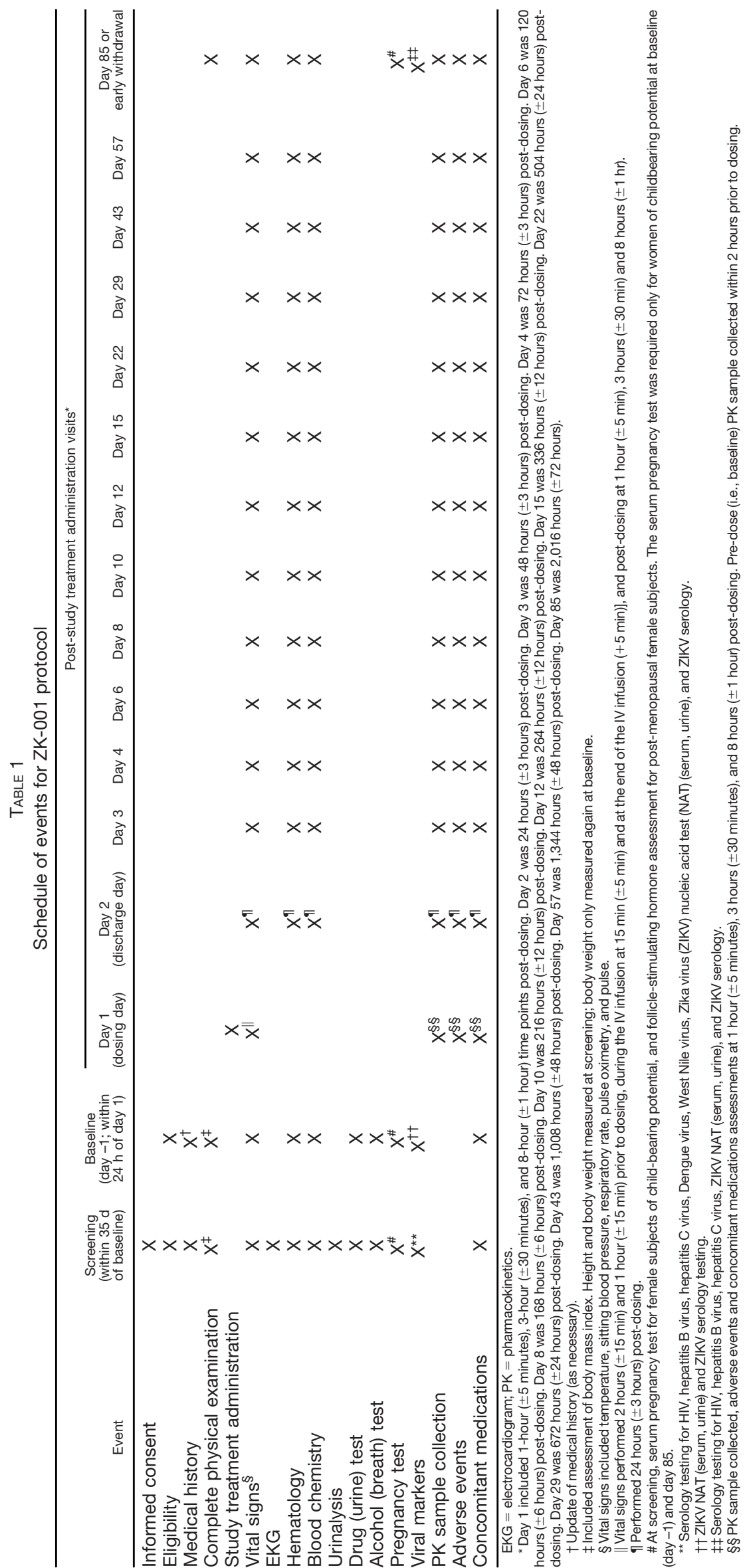


attachment to cells. Next, the sample and virus mixtures were washed from the cells and replaced with serum-free media containing $1 \%$ bovine serum albumin to allow cells to remain viable throughout a 3 -day incubation period. This incubation period allowed replication of the virus to provide a suitable level on virus-induced cytopathogenicity. Sample end point concentration was determined by interpolation of the signal against a standard curve with known levels of the ZIKV-Ig reference standard.

Statistical analysis. The sample size was consistent with a phase 1 first-in-human study and there was no formal sample size calculation. The safety analysis included the safety population that consisted of all subjects who received any amount of study treatment, and was based on the treatment received (ZIKV-lg or placebo). Descriptive statistics and changes from baseline in continuous clinical laboratory results and vital sign results, as well as frequency of clinically significant findings for clinical laboratory results, vital sign results, and physical examinations are presented by treatment and time point for each test. The PK population included all subjects who received ZIKV-Ig with an adequate number of PK samples (a suitable pre-dose sample and at least one measurable post-dose sample).

The PK parameters included area under the concentration-time curve (AUC) from time 0 to the last quantifiable concentration $\left(A \cup C_{0-t}\right), A \cup C$ from time 0 to day 7 $\left(A \cup C_{0-\text { day }} 7\right), A C_{0-t}$ plus the additional area extrapolated to infinity $\left(\mathrm{AUC}_{0-\infty}\right)$, the extrapolated area as a percentage of $\mathrm{AUC}_{0-\infty}\left(\mathrm{AUC}_{\%}\right.$ extrapolated $)$, the maximum observed concentration $\left(\mathrm{C}_{\text {max }}\right)$, the time at which $\mathrm{C}_{\max }$ occurs $\left(\mathrm{T}_{\text {max }}\right)$, the terminal elimination rate constant $\left(\lambda_{\mathrm{z}}\right)$, the apparent first-order terminal elimination half-life $\left(t_{1 / 2}\right)$, total body clearance following IV administration (CL), the volume of distribution following IV administration $\left(\mathrm{V}_{\mathrm{z}}\right)$, the adjusted $r^{2}$ value for $\lambda_{\mathrm{z}}$ regression, the number of points used in the $\lambda_{z}$ calculation, and the lower and upper range of times for points used in the $\lambda_{\mathrm{z}}$ calculation.

Serum concentration versus time data were analyzed by standard non-compartmental methods (i.e., linear trapezoidal method for consecutive time points with level or increasing concentrations and log-linear trapezoidal method for consecutive time points with decreasing concentrations with no interpolation). The terminal elimination rate constant, used to determine $t_{1 / 2}$, was calculated as the negative of the slope of the terminal portion of the observed serum concentration-time curve. In the non-compartmental analysis, concentrations of total antibody less than the lower limit of quantification were set to zero. Actual times and nonnominal times were used in the analysis. The PK parameters were calculated using non-compartmental analysis.

\section{RESULTS}

The study was conducted from June 27, 2018 to March 6, 2019. A total of 309 volunteers were screened for this study, of which 30 subjects were randomized and assigned sequentially to receive either ZIKV-Ig $(n=19)$ or placebo $(n=11)$ as illustrated in Figure 2. Excluded volunteers $(n=279)$ did not meet inclusion criteria or met exclusion criteria (screen failures), declined to participate (withdrew consent), or met screening criteria but were not enrolled $(n=21)$ (i.e., considered backup subjects).
In total, all 30 randomized subjects received their intended study drug and were included in the safety population. A total of 19 subjects received ZIKV-Ig and 11 subjects received placebo. Each subject received the total planned single-dose volume of $50.0 \mathrm{~mL}$, except one subject in the placebo group who received $49.9978 \mathrm{~mL}$ (99.9956\%) of the intended $50.0 \mathrm{~mL}$ dose. All subjects completed the infusion of the study drug with a mean duration of infusion of 32.7 minutes (range, 32-48 minutes).

All 19 subjects in the ZIKV-lg cohort completed the study, whereas 1 of 11 subjects (3.3\%) in the placebo cohort was discontinued from the study (lost to follow-up). All 30 subjects were included in the safety population, and 18 of 19 subjects who received ZIKV-Ig were included in the PK population. One subject was removed from PK analyses as an outlier. This subject had a much later and lower $\mathrm{C}_{\max }$ value, with an apparent absorption phase present. It was hypothesized the IV infiltration may have affected the $\mathrm{C}_{\max }$. For this subject, the infusion started at a $1-\mathrm{mL} / \mathrm{min}$ initial infusion rate that was increased to $2 \mathrm{~mL} / \mathrm{min}$ after 15 minutes (per protocol). Two minutes after the infusion rate increase, the infusion stopped because of the syringe pump high-pressure alarm. The infusion was restarted at a reduced infusion rate of $1 \mathrm{~mL} / \mathrm{min}$. Although this subject ultimately received the total $50.0-\mathrm{mL}$ dose, the total infusion duration was 48 minutes. The PI noted in the source notes that it was possible that some of the infusion volume had gone interstitial. There was a non-serious $A E$ reported as swelling in the right antecubital arm (preferred term, peripheral swelling) with mild intensity and not-related causality for this subject. The $\mathrm{AE}$ was monitored and was considered resolved the next day.

Of the 30 subjects enrolled, 22 (73.3\%) were men and $8(26.7 \%)$ were women, with a mean age of 35.84 years and a baseline body mass index of $24.5 \mathrm{~kg} / \mathrm{m}^{2}$. The majority of subjects were white (73.3\%), and all subjects had blood type O (86.7\% had type $\mathrm{O}+$ and $13.3 \%$ had type O-). Subject demographics and baseline characteristics are listed in Table 2.

Twenty of 30 subjects $(66.7 \%)$ reported 55 AEs. Of these, 12 subjects (63.2\%) were from the ZIKV-Ig group compared with eight subjects $(72.7 \%)$ from the placebo group. There were 51 AEs $(92.7 \%)$ considered mild in intensity. Four of the 55 AEs $(7.3 \%)$ in 4 of 30 subjects $(13.3 \%)$ were considered moderate in intensity, including two AEs in 2 of 19 subjects (10.5\%) in the ZIKV-Ig group (lower abdominal pain, streptococcal pharyngitis) and two AEs in 2 of 11 subjects (18.2\%) in the placebo group (abdominal abscess, back pain). None of these AEs were considered related to the study drug.

No deaths and no serious adverse events or severe AEs were observed during the study period in either the ZIKV-Ig or placebo study groups. No subject had drug discontinuation (e.g., infusion interruption with a decision not to restart and complete the infusion) as a result of AEs. One subject from the placebo group experienced a not-related AESI of rash reported 2 months after infusion. The mean and median changes from baseline for all hematology and blood chemistry parameters were minimal and similar between the ZIKVIg group and the placebo group. There were no related AEs resulting from abnormal vital signs or laboratory values, and no anomalous results were found. There were no clinically significant abnormal vital sign measurements in either treatment group during the study; however, an aspartate aminotransferase elevation (113 U/L) at day 10 in one subject in 


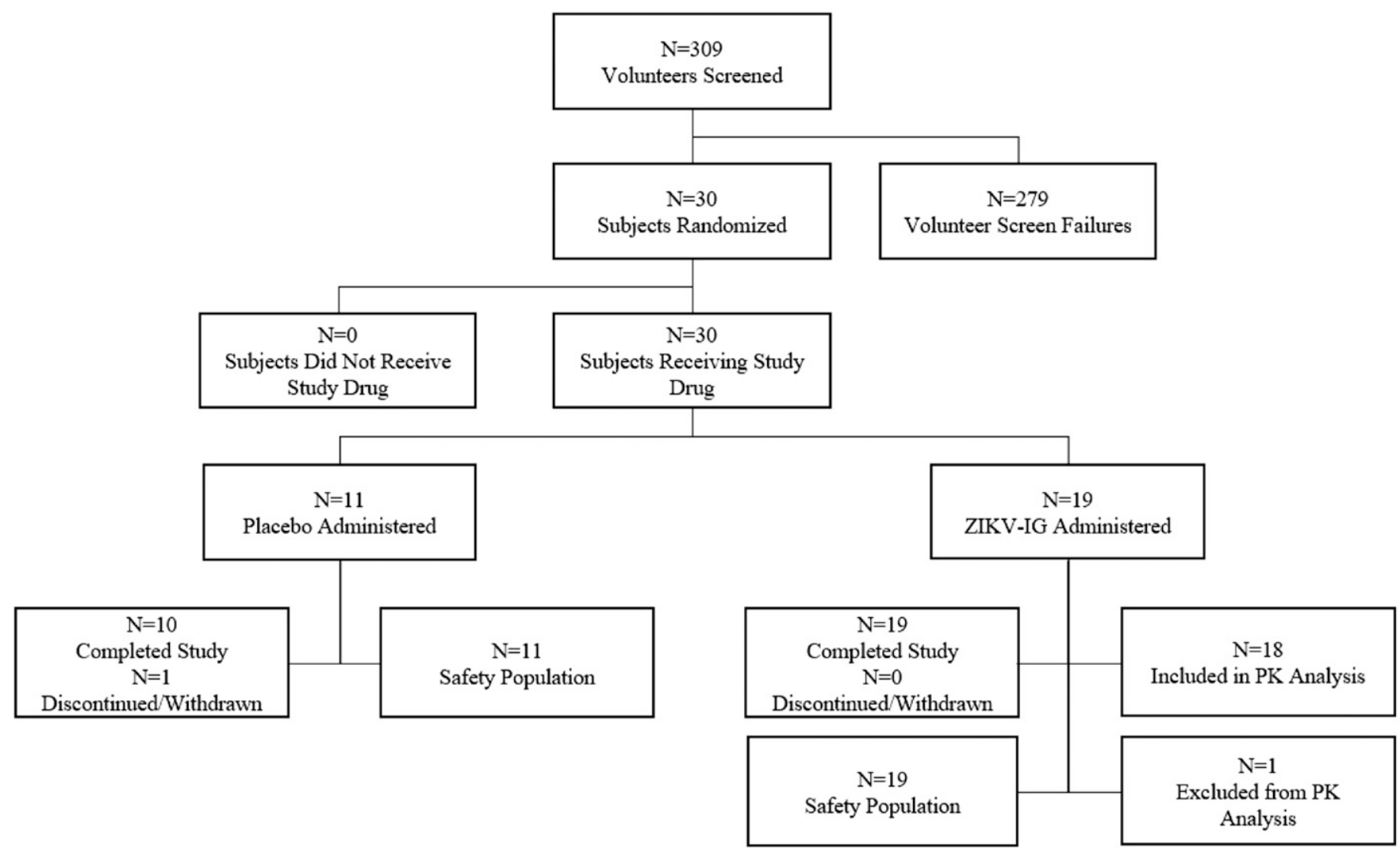

FIGURE 2. Disposition of volunteers. A total of 309 volunteers were screened for the ZK-001 study. Of these, 279 were screen failures. Thirty subjects met the study eligibility criteria, were randomized, and were administered either human anti-Zika virus immunoglobulin (ZIKV-lg) ( $n=19)$ or placebo $(n=11)$. All 19 subjects in the ZIKV-Ig cohort completed the study, whereas 1 of 11 subjects in the placebo cohort was discontinued from the study (lost to follow-up). All 30 subjects were included in the safety population, and 18 out of 19 subjects who received ZIKV-Ig were included in the pharmacokinetics (PK) population.

the ZIKV-Ig group was deemed clinically important by the PI. The PI assessed one subject in the ZIKV-Ig group as having a tender and enlarged submandibular lymph node at end-ofstudy day 85 , indicating it was clinically significant during a physical examination.

The most frequent AEs ( $>5 \%$ of study subjects) reported included the following: six headache events in five subjects (16.7\%), three events of dizziness in three subjects $(10.0 \%)$, and three events of nausea in two subjects $(6.7 \%)$. There were two events of fatigue, peripheral swelling, and nasal congestion in two subjects $(6.7 \%)$ each. There were also two events of increase aspartate aminotransferase and increased blood lactate dehydrogenase in two subjects $(6.7 \%)$ each. All the remaining reported AEs were reported once in one subjects (3.3\%) each, including dysgeusia, paresthesia, abdominal distension, lower abdominal pain, diarrhea, dry mouth, salivary hypersecretion, tongue pigmentation, catheter site hypoesthesia, feeling of body temperature change, influenza-like illness, vessel puncture site bruise, increased alanine aminotransferase, increased blood creatine phosphokinase, increased myoglobin blood, abdominal abscess, hordeolum, oral herpes, streptococcal pharyngitis, upper respiratory tract infection, viral upper respiratory tract infection, oropharyngeal pain, back pain, myalgia, menorrhagia, irregular menstruation, rash, skin lesion, lymphadenopathy, blurred vision, arthropod bite, hypervigilance, and hematuria.
Overall, the most reported related AEs (>5\%) in the safety population (ZIKV-lg and placebo) were headache (three subjects, 10\%) and dizziness (two subjects, 6.7\%). Other related AEs reported in one subject (3.3\%) each are listed in Table 3. A total of 12 AEs in eight subjects $(42.1 \%)$ were reported as related to ZIKV-Ig compared with five AEs in three subjects $(27.3 \%)$ considered as related to placebo. The most frequently reported related $\mathrm{AE}$ was headache in three (15.8\%) of the ZIKV-Ig-treated subjects; whereas no placebo subjects had treatment-related headache reported. In all three of these subjects, the headache was mild and resolved without treatment and with no sequelae. One subject in the ZIKV-Ig group had 4 of the 12 (33.3\%) AEs that the PI considered to be to be related to the drug, including dry mouth, metallic taste in mouth (dysgeusia), paresthesia (left arm), and diarrhea, all of which were mild and resolved without treatment and with no sequelae within 1 to 2 days of onset. Another subject had transient self-resolving mild nausea during the ZIKV-lg infusion, as well as myalgia (muscle pain) with onset occurring 5 days after ZIKV-lg infusion that resolved within 3 days after treatment with one dose of $600 \mathrm{mg}$ oral Ibuprofen.

PK data were available from pre-dose (i.e., baseline), collected within 2 hours prior to dosing, to end-of-study day 85 for all 19 evaluable subjects who received ZIKV-Ig. After IV administration of ZIKV-lg, the change in serum concentration of anti-ZIKV E-protein binding antibodies and neutralizing 
TABLE 2

Subject demographics and baseline characteristics

\begin{tabular}{|c|c|c|c|}
\hline Parameter & ZIKV-Ig $(n=19)$ & Placebo $(n=11)$ & Total $(N=30)$ \\
\hline \multicolumn{4}{|l|}{ Gender at birth, n (\%) } \\
\hline Male & $15(78.9)$ & $7(63.6)$ & $22(73.3)$ \\
\hline Female & $4(21.1)$ & $4(36.4)$ & $8(26.7)$ \\
\hline Childbearing potential, yes & $4(21.1)$ & $4(36.4)$ & $8(26.7)$ \\
\hline \multicolumn{4}{|l|}{ Age, y } \\
\hline Mean (SD) & $35.06(9.62)$ & $37.18(13.72)$ & $35.84(11.12)$ \\
\hline Median & 35.0 & 30.0 & 33.5 \\
\hline Min, $\max$ & $21.0,52.0$ & $23.0,55.0$ & $21.0,55.0$ \\
\hline \multicolumn{4}{|l|}{ Race, n (\%) } \\
\hline Asian & $3(15.8)$ & $2(18.2)$ & $5(16.7)$ \\
\hline Black or African American & $1(5.3)$ & $0(0)$ & $1(3.3)$ \\
\hline Other & $1(5.3)$ & $1(9.1)$ & $2(6.7)$ \\
\hline White & $14(73.7)$ & $8(72.7)$ & $22(73.3)$ \\
\hline \multicolumn{4}{|l|}{ Ethnicity, n (\%) } \\
\hline Hispanic or Latino & $1(5.3)$ & $3(27.3)$ & $4(13.3)$ \\
\hline Not Hispanic or Latino & $18(94.7)$ & $8(72.7)$ & $26(86.7)$ \\
\hline \multicolumn{4}{|l|}{ Height (m) } \\
\hline Mean (SD) & $174.22(7.86)$ & $172.54(9.08)$ & $173.60(8.20)$ \\
\hline Median & 175.2 & 170.0 & 174.3 \\
\hline Min, $\max$ & $160.1,186.5$ & $156.5,186.8$ & $156.5,186.8$ \\
\hline \multicolumn{4}{|l|}{ Baseline weight $(\mathrm{kg})$} \\
\hline Mean (SD) & $75.52(11.78)$ & $72.10(14.26)$ & $74.26(12.60)$ \\
\hline Median & 75.0 & 71.0 & 74.0 \\
\hline Min, $\max$ & $52.0,102.0$ & $54.0,98.0$ & $52.0,102.0$ \\
\hline \multicolumn{4}{|c|}{ Baseline body mass index $\left(\mathrm{kg} / \mathrm{m}^{2}\right)$} \\
\hline Mean (SD) & $24.74(2.38)$ & $24.10(3.42)$ & $24.50(2.76)$ \\
\hline Median & 24.1 & 23.8 & 24.1 \\
\hline Min, max & $19.9,30.0$ & $17.6,29.9$ & $17.6,30.0$ \\
\hline \multicolumn{4}{|l|}{ Blood type, n (\%) } \\
\hline $\mathrm{O}+$ & $17(89.5)$ & $9(81.8)$ & $26(86.7)$ \\
\hline O- & $2(10.5)$ & 2 (18.2) & $4(13.3)$ \\
\hline
\end{tabular}

antibodies over time both followed a biphasic disposition, with an initial rapid distribution phase followed by a slow elimination phase (Figure 3).

The summary of ZIKV-lg PK characteristics of anti-ZIKV E-protein binding antibodies in subjects receiving a single dose, and the PK parameters of ZIKV-Ig based on serum concentrations of ZIKV neutralizing antibodies over time are summarized in Table 4.

\section{DISCUSSION}

Currently, there is no approved therapeutic or licensed ZIKV vaccines to prevent ZIKV infection or disease, including

TABLE 3

Adverse events assessed as related to study drug by the Medical Dictionary for Regulatory Activities system organ class and preferred term (safety population)

\begin{tabular}{|c|c|c|c|}
\hline System organ class preferred term & ZIKV-Ig $(n=19) ; \mathrm{m}, \mathrm{n}\left(\%^{*}\right)$ & Placebo $(n=11) ; \mathrm{m}, \mathrm{n}\left(\%^{\star}\right)$ & Total $(N=30) ; \mathrm{m}, \mathrm{n}\left(\%^{\star}\right)$ \\
\hline No. of related adverse events and subjects with related adverse events & $12,8(42.1)$ & $5,3(27.3)$ & $17,11(36.7)$ \\
\hline Nervous system disorders & $6,5(26.3)$ & $1,1(9.1)$ & $7,6(20)$ \\
\hline Headache & $3,3(15.8)$ & $0,0(0)$ & $3,3(10)$ \\
\hline Dizziness & $1,1(5.3)$ & $1,1(9.1)$ & $2,2(6.7)$ \\
\hline Dysgeusia & $1,1(5.3)$ & $0,0(0)$ & $1,1(3.3)$ \\
\hline Paresthesia & $1,1(5.3)$ & $0,0(0)$ & 1,1 (3.3) \\
\hline Gastrointestinal disorders & $3,2(10.5)$ & $1,1(9.1)$ & $4,3(10)$ \\
\hline Diarrhea & $1,1(5.3)$ & $0,0(0)$ & 1,1 (3.3) \\
\hline Dry mouth & $1,1(5.3)$ & $0,0(0)$ & $1,1(3.3)$ \\
\hline Nausea & $1,1(5.3)$ & $0,0(0)$ & 1,1 (3.3) \\
\hline Salivary hypersecretion & $0,0(0)$ & $1,1(9.1)$ & $1,1(3.3)$ \\
\hline General disorders and administration site conditions & $1,1(5.3)$ & $2,2(18.2)$ & $3,3(10)$ \\
\hline Catheter site hypoesthesia & $1,1(5.3)$ & $0,0(0)$ & 1,1 (3.3) \\
\hline Fatigue & $0,0(0)$ & $1,1(9.1)$ & $1,1(3.3)$ \\
\hline Feeling of body temperature change & $0,0(0)$ & $1,1(9.1)$ & 1,1 (3.3) \\
\hline Musculoskeletal and connective tissue disorders & $1,1(5.3)$ & $0,0(0)$ & $1,1(3.3)$ \\
\hline Myalgia & $1,1(5.3)$ & $0,0(0)$ & $1,1(3.3)$ \\
\hline Psychiatric disorders & $0,0(0)$ & $1,1(9.1)$ & $1,1(3.3)$ \\
\hline Hypervigilance & $0,0(0)$ & $1,1(9.1)$ & $1,1(3.3)$ \\
\hline Skin and subcutaneous tissue disorders & $1,1(5.3)$ & $0,0(0)$ & 1,1 (3.3) \\
\hline Skin lesion & $1,1(5.3)$ & $0,0(0)$ & $1,1(3.3)$ \\
\hline
\end{tabular}

${ }^{*}(\mathrm{n} / n) \times 100$. Subjects with more than one event in a category are counted once in each category. 

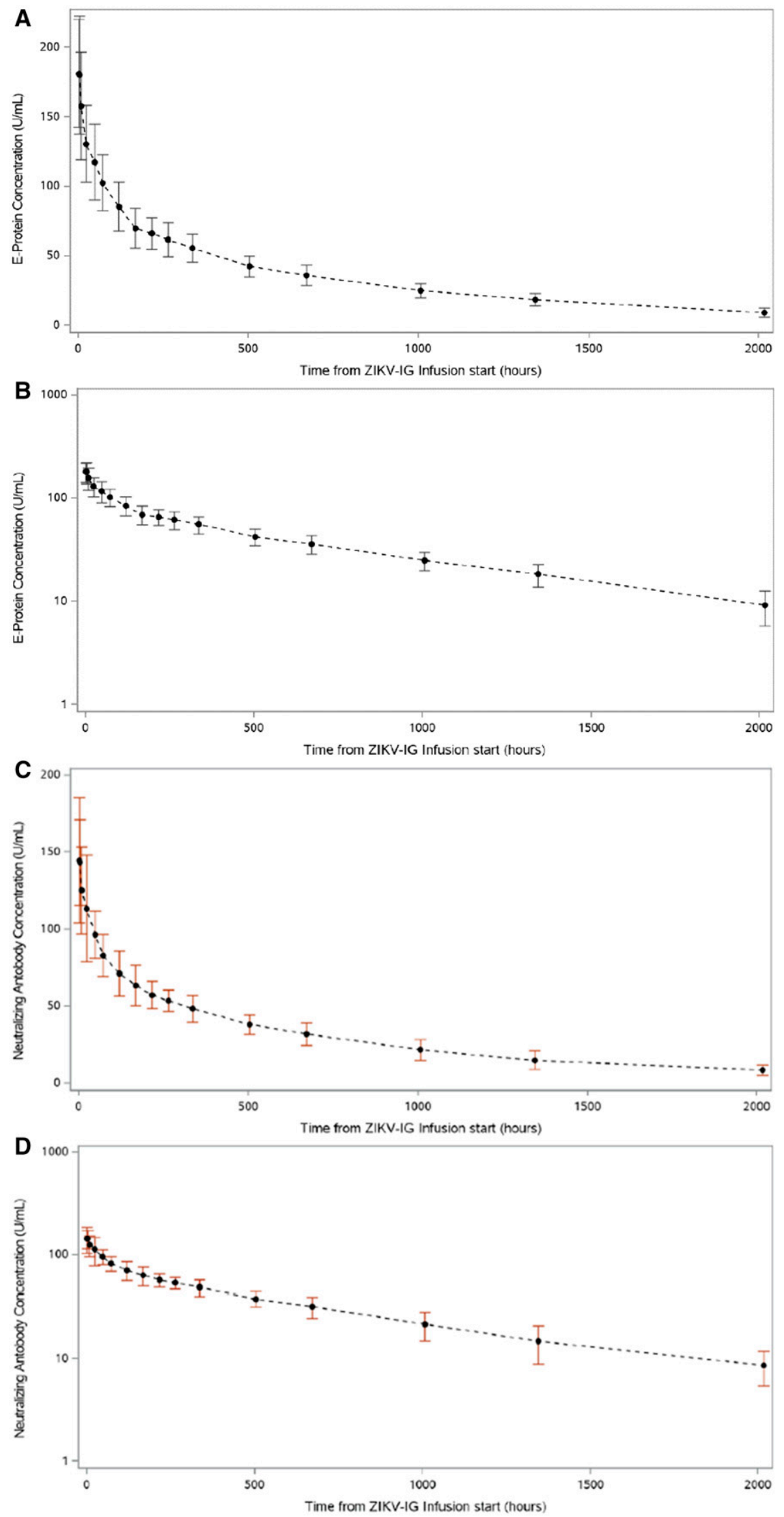

FIGURE 3. Mean ( \pm SD) concentration-time profiles for anti-Zika virus (ZIKV) E-protein binding antibodies on a linear scale (panel A) and a semilogarithmic scale (panel B) [pharmacokinetic (PK) population). Mean ( \pm SD) concentration-time profiles for anti-ZIKV neutralizing antibodies on a linear scale (panel C) and a semi-logarithmic scales (panel D) (PK population). This figure appears in color at www.ajtmh.org. 
TABLE 4

Pharmacokinetic characteristics of Zika virus E-protein binding and neutralizing antibodies (pharmacokinetics population)

\begin{tabular}{|c|c|c|c|c|}
\hline PK parameter & Unit & Statistic & $\begin{array}{l}\text { E-protein binding antibodies } \\
\text { ZIKV-lg }\left(n=181^{*}\right)\end{array}$ & $\begin{array}{l}\text { Neutralizing antibodies } \\
\text { ZIKV-lg }\left(n=181^{*}\right)\end{array}$ \\
\hline \multirow[t]{5}{*}{$\mathrm{AUC}_{0-\mathrm{t}}$} & $\mathrm{h} \times \mathrm{U} / \mathrm{mL}$ & Geometric mean & 67,221 & $50,235.64$ \\
\hline & & Geometric CV\% & 18.8 & 21.0 \\
\hline & & Mean (SD) & $68,319(12,596)$ & $51,299.88(11,281.39)$ \\
\hline & & Median & 67,700 & $49,000.2$ \\
\hline & & Min; max & 46,$431 ; 94,737$ & 34,583.0; 83,312.6 \\
\hline \multirow[t]{5}{*}{$\mathrm{AUC}_{0-7}$} & $\mathrm{~h} \times \mathrm{U} / \mathrm{mL}$ & Geometric mean & 14,986 & $12,456.08$ \\
\hline & & Geometric CV\% & 25.8 & 23.6 \\
\hline & & Mean (SD) & $15,466(4,198.6)$ & $12,773.22(2,912.79)$ \\
\hline & & Median & 14,706 & $12,235.1$ \\
\hline & & Min; max & 9,$629 ; 27,184$ & $8,227.5 ; 17,803.6$ \\
\hline \multirow[t]{5}{*}{$\mathrm{AUC}_{0-\infty}$} & $\mathrm{h} \times \mathrm{U} / \mathrm{mL}$ & Geometric mean & 77,224 & $67,954.72$ \\
\hline & & Geometric CV\% & 17.9 & 23.5 \\
\hline & & Mean (SD) & $78,374(13,832)$ & $69,732.01(16,704.13)$ \\
\hline & & Median & 77,798 & $66,741.2$ \\
\hline & & Min; max & 55,$892 ; 103,130$ & $45,422.4 ; 107,532.9$ \\
\hline \multirow{5}{*}{ AUC $\%$ extrapolated } & $\%$ & Geometric mean & 12.27 & 24.82 \\
\hline & & Geometric CV\% & 33.04 & 25.0 \\
\hline & & Mean (SD) & 12.87 (3.99) & $25.63(7.64)$ \\
\hline & & Median & 13.1 & 23.6 \\
\hline & & Min; max & $7.1 ; 19.3$ & $18.3 ; 50.3$ \\
\hline \multirow[t]{5}{*}{$\mathrm{C}_{\max }$} & $\mathrm{U} / \mathrm{mL}$ & Geometric mean & 182.3 & 150.15 \\
\hline & & Geometric CV\% & 21.3 & 26.3 \\
\hline & & Mean (SD) & $186.3(41.4)$ & $155.02(41.26)$ \\
\hline & & Median & 183 & 146.0 \\
\hline & & Min; max & $128 ; 303$ & $95.6 ; 256.9$ \\
\hline \multirow[t]{3}{*}{$t_{\max }$} & $\mathrm{h}$ & Mean (SD) & $2.3(1.0)$ & $3.83(5.26)$ \\
\hline & & Median & 2 & 3.5 \\
\hline & & Min; max & $2 ; 4$ & $1.6 ; 24.5$ \\
\hline \multirow[t]{5}{*}{$\lambda_{\mathrm{z}}$} & $/ \mathrm{h}$ & Geometric mean & 0.00103 & 0.00111 \\
\hline & & Geometric CV\% & 16.38765 & 43.4 \\
\hline & & Mean (SD) & $0.00104(0.00017)$ & $0.00120(0.00045)$ \\
\hline & & Median & 0.0010 & 0.0012 \\
\hline & & Min; max & $0.0008 ; 0.0014$ & $0.0004 ; 0.0023$ \\
\hline \multirow{5}{*}{$r^{2}$ Value } & Proportion & Geometric mean & 0.99495 & 0.94680 \\
\hline & & Geometric CV\% & 1.02524 & 5.8 \\
\hline & & Mean (SD) & $0.99500(0.01004)$ & $0.94823(0.05203)$ \\
\hline & & Median & 0.9983 & 0.9545 \\
\hline & & Min; max & $0.9577 ; 1.0000$ & $0.7923 ; 0.9976$ \\
\hline \multirow{5}{*}{ No. of points used in $\lambda_{z}$ calculation } & Count & Geometric mean & 4.1 & 5.70 \\
\hline & & Geometric CV\% & 45.5 & 52.2 \\
\hline & & Mean (SD) & $4.6(2.4)$ & 6.39 (3.18) \\
\hline & & Median & 4 & 5.5 \\
\hline & & Min; $\max$ & $3 ; 10$ & $3.0 ; 13.0$ \\
\hline \multirow[t]{5}{*}{$t_{1 / 2}$} & $\mathrm{~h}$ & Geometric mean & 674.7 & 624.76 \\
\hline & & Geometric CV\% & 16.4 & 43.4 \\
\hline & & Mean (SD) & $683.1(110.8)$ & $683.34(340.23)$ \\
\hline & & Median & 686 & 586.6 \\
\hline & & Min; $\max$ & 508; 889 & $304.2 ; 1,772.7$ \\
\hline \multirow[t]{5}{*}{ CL } & $\mathrm{mL} / \mathrm{h}$ & Geometric mean & 6.871 & 7.808 \\
\hline & & Geometric CV\% & 17.9 & 23.5 \\
\hline & & Mean (SD) & $7.0(1.3)$ & $8.01(1.84)$ \\
\hline & & Median & 7 & 8.0 \\
\hline & & Min; $\max$ & $5 ; 9$ & $4.9 ; 11.7$ \\
\hline \multirow[t]{5}{*}{$V_{z}$} & $\mathrm{~mL}$ & Geometric mean & $6,687.7$ & $7,037.73$ \\
\hline & & Geometric CV\% & 20.1 & 26.4 \\
\hline & & Mean (SD) & $6,809.9(1,299.2)$ & $7,286.78(2,181.80)$ \\
\hline & & Median & 6,865 & $6,570.1$ \\
\hline & & Min; max & 4,318; 9,426 & $5,126.3 ; 13,141.3$ \\
\hline \multirow[t]{3}{*}{$\lambda_{z}$ lower limit } & $\mathrm{h}$ & Mean (SD) & 705.7 (337.4) & 324.78 (243.12) \\
\hline & & Median & 674 & 276.2 \\
\hline & & Min; max & $118 ; 1,055$ & $8.6 ; 672.9$ \\
\hline \multirow{3}{*}{$\lambda_{z}$ upper limit } & $\mathrm{h}$ & Mean (SD) & $1,937.8(216.7)$ & 1,267.20 (355.54) \\
\hline & & Median & 2,016 & $1,344.1$ \\
\hline & & Min; max & 1,$345 ; 2,041$ & $672.4 ; 2,016.2$ \\
\hline
\end{tabular}

AUC = area under the concentration-time curve; CV = coefficient of variation; max = maximum; min = minimum; PK = pharmacokinetics; ZIKV-lg = human anti-Zika virus immunoglobulin; $A \cup C_{0-t}=$ area under the concentration-time curve from time 0 to the last quantifiable concentration; $A \cup C_{0 \text {-day } 7}=$ area under the concentration-time curve from time 0 to day $7 ; A C_{0-\infty}=A \cup C_{0-t}$ plus the additional area extrapolated to infinity; $A \cup C_{\%}$ extrapolated $=$ extrapolated area as a percentage of $A \cup C_{0-\infty} ; C_{\max }=$ maximum observed concentration; $T_{\max }=$ time at which $C_{\text {max }}$ Occurs; $\lambda_{z}=$ terminal elimination rate constant; $t_{1 / 2}=$ apparent first order terminal elimination half-life; $C L=$ total body clearance following IV administration; $V_{z}=$ volume of distribution following IV administration.

*One subject was removed from PK analyses as an outlier. 
in populations at risk, such as pregnant women. Passive immunization with an antibody therapeutic such as ZIKV-Ig could provide protection against ZIKV infection (with regular dosing to maintain ZIKV antibody levels during pregnancy). $\mathrm{EBCl}$ is developing ZIKV-lg, a human hyperimmune product of purified IgG fraction of human plasma containing antibodies to ZIKV as an intervention to ZIKV to address this unmet need. ZIKV-Ig was manufactured using the same hyperimmune manufacturing platform process as the one used to manufacture U.S. FDA-licensed products.

The selection of the ZIKV-Ig dose level of $50.0 \mathrm{~mL}$ ZIKV-lg $(\sim 0.1 \mathrm{~g} / \mathrm{kg})$, containing a total of $4.65 \mathrm{~g}$ protein, was estimated to yield protective levels of ZIKV antibodies based on immune-correlated data from ZIKV infection studies ${ }^{18,19}$ in nonhuman primates (NHPs) and is within the efficacious ZIKV-lg doses $(0.05-0.4 \mathrm{~g} / \mathrm{kg})$ evaluated in the mouse model of ZIKV infection. ${ }^{20}$ The selected $50.0-\mathrm{mL}$ dose of ZIKV-Ig falls within the range of doses used for the other products manufactured with EBCl's hyperimmune platform and is considered a low Ig IV dose because standard Ig IV doses range from 0.2 to $1 \mathrm{~g} / \mathrm{kg}$.

Igs are normal constituents of blood, and they are used at physiological levels without creating pharmacological and toxicological active metabolites. ${ }^{21}$ The safety profile for $\mathrm{Ig}$ products is well established as a result of a long history of their use in clinical practice for a range of medical conditions. ${ }^{17,18,22}$ The most common types of AEs related to IV Ig and hyperimmune products are non-anaphylactic infusion reactions (self-limiting), such as abdominal or back pain, fever, headache, chills, rash, fatigue, nausea, or vomiting. The incidence of adverse reactions associated with IV Ig's is in the range of $1 \%$ to $15 \%$, but is typically $\leq 5 \%$. AESI such as hypersensitivity reactions, renal dysfunction/failure, aseptic meningitis syndrome, hemolysis, transfusion-related acute lung injury, and thrombotic events have been reported infrequently with IV Ig. ZIKV-Ig was safe and well tolerated in healthy adult subjects with blood types $\mathrm{O}+$ and $\mathrm{O}-$. Related AEs that were reported more frequently in the ZIKV-Ig group compared with the placebo group included headaches. These were reported only in ZIKV-Ig group, which is an expected type of related $A E$ (i.e., adverse drug reaction) for a human IV Ig product. There were no AESI reported in the 19 subjects administered ZIKV-lg in our study, suggesting a similar safety profile to other IV Ig products.

The PK parameters were characterized with a validated binding assay (using a recombinant ZIKV E-protein antigen) and were confirmed further by a functional ZIKV neutralization assay. The $x$ Celligence functional neutralization assay results were also similar to the results obtained by the MAGPIX binding assay, although they were slightly lower, as expected, because neutralizing antibodies are a subset of binding antibodies. Overall, both assays used to analyze the PK concentrations in our study demonstrated similar PK trends for ZIKV-Ig. The PK parameters for ZIKV-Ig were similar to PK parameters of other human IV Ig products manufactured using EBCl's hyperimmune platform and other commercial IV Ig products. Although the doses and protein concentrations vary by products and indication, the drug product is primarily purified human $\lg G$ and is expected to have a similar half-life to other commercial products (e.g., (HepaGam B ${ }^{\circledR}$ (Hepatitis B Immune Globulin Intravenous [Human]), Emergent BioSolutions, Winnipeg, Canada,
VariZIG $^{\circledR}$ (Varicella Zoster Immune Globulin [Human)]; Emergent BioSolutions, Winnipeg, Canada, VIGIV ${ }^{\circledR}$ Vaccinia Immune Globulin Intravenous (Human); Emergent BioSolutions, Winnipeg, Canada, ANTHRASIL ${ }^{\circledR}$ (Anthrax Immune Globulin Intravenous [Human]; Emergent BioSolutions, Winnipeg, Canada).). As demonstrated in our study, ZIKV-Ig had a calculated mean half-life of 28.1 days, which is in the range of the expected half-life for this product class (i.e., 21-30 days). The $\mathrm{C}_{\max }, \mathrm{T}_{\max }$, and AUC of ZIKV-Ig were also consistent with those of human-derived IgG products.

In conclusion, ZIKV-Ig appears safe and was well-tolerated in healthy adult subjects with blood types $\mathrm{O}+$ and $\mathrm{O}-$, and had PK parameters consistent with the expected PK of other commercially available human hyperimmune products.

Received December 12, 2020. Accepted for publication August 6, 2021.

Published online October 4, 2021.

Note: Supplemental appendices appear at www.ajtmh.org.

Acknowledgments: We thank all the volunteers who were screened and subjects who participated in the study. Thanks to the staff at INC Research/Syneos Health; Vadim Dreyzin, MD; Michael McDonnell, MD; Stiris Research Inc.; Nuventra Pharma Sciences Inc.; and the Advarra Institutional Review Board Canadian Panel. We also thank Emergent personnel Poly Shinkarik, Allan Chand, Douglas Barker, Shantha Kodihalli, Hongyu Qiu, Monica Viteri, Derek Toth, Trevor Carnelley, Samantha Erichsen, Karolyn Gale, Mandy Gibson, Dana Kowalsky, Steve McGregor, Tim Babinchak, Christine Hall, and Paul-André de Lame (Anabase International Corp). This manuscript is dedicated in memory of Zavier Chand. The authors confirm that all ongoing and related trials for this drug/intervention are registered (\#NCT03624946). This trial is registered at ClinicalTrials.gov https:// clinicaltrials.gov/ct2/show/NCT03624946?term $=z k-001 \&$ draw $=2 \&$ rank=1.

Financial support: This work was funded by Emergent BioSolutions Inc.

Disclosure: ANTHRASIL ${ }^{\circledR}$ (Anthrax Immune Globulin Intravenous [Human]; Emergent BioSolutions, Winnipeg, Canada, CNJ-016 ${ }^{\text {TM }}$ (Vaccinia Immune Globulin Intravenous (Human) [VIGIV]); Emergent BioSolutions, Winnipeg, Canada, and any and all Emergent BioSolutions Inc. brands, products, services, and feature names, logos, and slogans are trademarks or registered trademarks of Emergent BioSolutions Inc. or its subsidiaries in the United States or other countries. All other brands, products, services, and feature names or trademarks are the property of their respective owners.

Authors' addresses: Jane White, Emergent BioSolutions Inc., Gaithersburg, MD, E-mail: whitej@ebsi.com. Priya Tunga, Deborah M. Anderson, Ken Iledan, Tobi Loreth, Geraldine S. Parrera, Hugo Astacio, Bojan Drobic, and Jason S. Richardson, Emergent BioSolutions Canada Inc., Winnipeg, Manitoba, Canada, E-mails: tungap@ebsi.com, andersond@ebsi.com, iledank@ebsi.com, loretht@ ebsi.com, gparrera@ebsi.com, hastacio@ebsi.com, bdrobic@ebsi. com, and jrichardson@ebsi.com.

This is an open-access article distributed under the terms of the Creative Commons Attribution (CC-BY) License, which permits unrestricted use, distribution, and reproduction in any medium, provided the original author and source are credited.

\section{REFERENCES}

1. Coelho FC et al., 2016. Higher incidence of Zika in adult women than adult men in Rio de Janeiro suggests a significant contribution of sexual transmission from men to women. Int $\mathrm{J}$ Infect Dis 51: 128-132.

2. Hennessey M, Fischer M, Staples JE, 2016. Zika virus spreads to new areas: region of the Americas, May 2015-January 2016. MMWR Morb Mortal Wkly Rep 65: 55-58. 
3. WHO, 2018. Zika Virus. Geneva, Switzerland: World Health Organization. Available at: https://www.who.int/news-room/ fact-sheets/detail/zika-virus. Accessed June 17, 2020.

4. WHO, 2019. Countries and Territories with Current or Previous Zika Virus Transmission. Geneva, Switzerland: World Health Organization. Available at: https://www.who.int/emergencies/ diseases/zika/countries-with-zika-and-vectors-table.pdf. Accessed June 29, 2020.

5. WHO, 2019. World Health Organization Zika Epidemiology Update: July 2019. Geneva, Switzerland: World Health Organization. Available at: https://www.who.int/emergencies/ diseases/zika/zika-epidemiology-update-july-2019.pdf?ua=1. Accessed June 29, 2020.

6. Arias A, Torres-Tobar L, Hernandez G, Paipilla D, Palacios E, Torres Y, Duran J, Ugarte US, Ardila-Sierra A, Castellanos G, 2017. Guillain-Barre syndrome in patients with a recent history of Zika in Cucuta, Colombia: a descriptive case series of 19 patients from December 2015 to March 2016. J Crit Care 37: 19-23.

7. Dirlikov E et al., 2016. Guillain-Barre syndrome during ongoing Zika virus transmission: Puerto Rico, January 1-July 31, 2016. MMWR Morb Mortal Wkly Rep 65: 910-914.

8. Ritter JM, Martines RB, Zaki SR, 2017. Zika virus: pathology from the pandemic. Arch Pathol Lab Med 141: 49-59.

9. Johansson MA, Mier-y-Teran-Romero L, Reefhuis J, Gilboa SM, Hills SL, 2016. Zika and the risk of microcephaly. N Engl $J$ Med 375: 1-4.

10. Kleber de Oliveira W, Cortez-Escalante J, De Oliveira WT, do Carmo GM, Henriques CM, Coelho GE, Araujo de Franca GV, 2016. Increase in reported prevalence of microcephaly in infants born to women living in areas with confirmed Zika virus transmission during the first trimester of pregnancy: Brazil, 2015. MMWR Morb Mortal Wkly Rep 65: 242-247.

11. Brasil $P$ et al., 2016. Zika virus infection in pregnant women in Rio de Janeiro. N Engl J Med 375: 2321-2334.
12. Ventura CV et al., 2016. Risk factors associated with the ophthalmoscopic findings identified in infants with presumed Zika virus congenital infection. JAMA Ophthalmol 134: 912-918.

13. Rasmussen SA, Jamieson DJ, Honein MA, Petersen LR, 2016. Zika virus and birth defects: reviewing the evidence for causality. N Engl J Med 374: 1981-1987.

14. Moore CA et al., 2017. Characterizing the pattern of anomalies in congenital Zika syndrome for pediatric clinicians. JAMA Pediatr 171: 288-295.

15. Shapiro-Mendoza CK et al., 2017. Pregnancy outcomes after maternal Zika virus infection during pregnancy: U.S. territories, January 1, 2016-April 25, 2017. MMWR Morb Mortal Wkly Rep 66: 615-621.

16. Tabata T, Petitt M, Puerta-Guardo H, Michlmayr D, Wang C, Fang-Hoover J, Harris E, Pereira L, 2016. Zika virus targets different primary human placental cells, suggesting two routes for vertical transmission. Cell Host Microbe 20: 155-166.

17. Coyne CB, Lazear HM, 2016. Zika virus: reigniting the TORCH. Nat Rev Microbiol 14: 707-715.

18. Aliota MT et al., 2016. Heterologous protection against Asian Zika virus challenge in Rhesus macaques. PLoS Negl Trop Dis 10: e0005168.

19. Chiu CY et al., 2017. Experimental Zika virus inoculation in a New World monkey model reproduces key features of the human infection. Sci Rep 7: 17126.

20. Branche E et al., 2019. Human polyclonal antibodies prevent lethal Zika virus infection in mice. Sci Rep 9: 9857.

21. Kurz M, 2008. Developing therapeutic immunoglobulins: European regulatory perspectives and implications. BioDrugs 22: 145-160.

22. U.S. Food and Drug Administration, 2020. Immune Globulins. Available at: https://www.fda.gov/vaccines-blood-biologics/ approved-blood-products/immune-globulins. Accessed March 4, 2021. 